\title{
Electrospun Fibers of Enteric Polymer for Controlled Drug Delivery
}

\author{
Fábia F. P. da Costa, ${ }^{1}$ Evando S. Araújo, ${ }^{1,2}$ \\ Marcio L. F. Nascimento, ${ }^{2}$ and Helinando P. de Oliveira ${ }^{1}$ \\ ${ }^{1}$ Instituto de Pesquisa em Ciência dos Materiais, Universidade Federal do Vale do São Francisco, 48920-310 Juazeiro, BA, Brazil \\ ${ }^{2}$ Programa de Pós-Graduação em Engenharia Industrial, Escola Politécnica, Universidade Federal da Bahia, \\ 40210-630 Salvador, BA, Brazil
}

Correspondence should be addressed to Helinando P. de Oliveira; helinando@gmail.com

Received 24 November 2014; Revised 19 December 2014; Accepted 3 March 2015

Academic Editor: Pornsak Sriamornsak

Copyright (C) 2015 Fábia F. P. da Costa et al. This is an open access article distributed under the Creative Commons Attribution License, which permits unrestricted use, distribution, and reproduction in any medium, provided the original work is properly cited.

The production of electrospun fibers of enteric polymer for controlled delivery of drugs represents a simple and low cost procedure with promising advantages relative to the longer therapeutic window provided by cylindrical geometry in association with intrinsic properties of $\mathrm{pH}$-dependent drug carriers. In this work, we have explored the incorporation of additives (block copolymers of poly(ethylene)-b-poly(ethylene oxide)) into matrix of Eudragit L-100 and the effective action of hybrid composites on delivery of nifedipine, providing improvement in the overall process of controlled release of loaded drug.

\section{Introduction}

The development of new strategies for controlled drug delivery has been progressively considered as an important tool for improvement in the drug activity at local target. Typically, the transit time of fluid and $\mathrm{pH}$ value at human gastrointestinal tract present a typical behavior in which increasing $\mathrm{pH}$ is established from stomach ( $\mathrm{pH}$ 1-2) to small intestine (upper part, duodenum, $\mathrm{pH} 4.5$ ) and jejunum and ileum (lower part of small intestine, $\mathrm{pH}$ 6.4) and reaches a value of 7.0 at colon [1].

The protonation provided by stomach (low $\mathrm{pH}$ region) improves the water solubility of drug while the high $\mathrm{pH}$ at intestine induces deprotonation and subsequent decrease in the solubility of drug. The encapsulation of drugs by enteric coating contributes with a complementary behavior: at low $\mathrm{pH}$, the solubility of enteric coating is negligible, provoking a strong reduction in the permeability of drug along the coating. At high $\mathrm{pH}$, the enteric coating becomes watersoluble in association with reduction in the drug solubility. As a consequence, the profile of drug release tends to be $\mathrm{pH}$ independent [2].
Colonic delivery systems [3] introduce interesting advantages relative to the total dose of drug in order to mitigate harmful effects, typically associated with oral administration. The protection of drug provided by $\mathrm{pH}$-dependent coating polymers contributes with controlled release at specific $\mathrm{pH}$. Eudragit L-100 (EDGT) is a common enteric coating composed of anionic blocks of metacrylic acid and methyl metacrylate with ratio of acid to ester of $1: 1$ which is insoluble in acidic condition and becomes soluble at pH 6 [4].

The encapsulation of drugs in the core of enteric polymers has been explored for oral delivery of insulin [5] and in the treatment of diseases such as ulcerative colitis [6] and applied in formulations for intestine, buccal, sublingual, transdermal, gene, and vaginal delivery. In this direction, the control in the diffusion degree of drug along polymeric coating at specific $\mathrm{pH}$ characterizes an important parameter for controlled release of drug at specific target. We have previously explored in our group the basic mechanisms related to $\mathrm{pH}$ dependent response of polymers [7] and controlled release of metronidazole encapsulated in Eudragit L-100 [8].

In addition to conventional methods for encapsulation (spherical microparticles of enteric polymers), the use of 
alternative techniques for production of new nanoscale morphologies for controlled release represents an emerging theme in the literature $[9,10]$. The development of cylindrical geometry for drug carrier introduces important properties for overall controlled release process such as longer therapeutic window and has been progressively considered as an additional application for electrospun fibers $[9,10]$.

The electrospinning technique, a straightforward procedure applied in the production of nanomaterials, requires a basic experimental setup composed by a high voltage source (in order of $15 \mathrm{kV}$ ) which provides high electrical field between the dip of a needle and a grounded target at few centimeters from ejection of charged jet [11].

Connected to the needle, a compartment with hydrogel at fixed pressure provides a regular flux of droplets in the absence of electrical field. At high voltage condition, charge accumulation takes place on depressed droplet and provokes the ejection of fibers according to Taylor's cone. The surface tension and diameter of resulting droplet have been considered critical parameters in the reduction of density of beads (imperfections) of resulting net, since the control in the relative concentration of coating polymer during electrospinning affects the diameter of resulting fibers and contributes with elevation in the transit time of drug along the body.

The application of electrospun fibers for drug release introduces interesting advantages relative to the incorporation of different active agents in polymeric template by electrospinning technique. The improvement in the bioavailability of drug is associated with minimization in side effects (nausea, diarrhea, and abdominal pain). In this direction, electrospun fibers have been progressively applied in tissue engineering, wound dressing, and drug delivery $[9,10]$. The potential for specific application has been attributed to the high surface to volume ratio, small diameter, and high porosity, which contributes with improvement in the adsorption of water poorly soluble drugs [9, 12, 13].

$\mathrm{Yu}$ et al. [14] reported that electrospun fibers of Eudragit series are typical flat materials with a characteristic disadvantage relative to the initial burst release effect for drug delivery applications. This process is typically circumvented by development of core-shell structures (coaxial electrospinning technique).

On the other side, the incorporation of drug into closedcell systems [15] such as polymeric micelles of block copolymers of poly(ethylene)-b-poly(ethylene oxide) provides adequate entrapment of poorly water-soluble drugs in polymeric core of nanostructures. Additional hydrogen bonds available on PEO portion improve the protection of drug against protein adsorption and cellular adhesion [16]. Resulting PEO corona effects increase the blood circulation time and result in the prolonged action of drug disposed in a stabilizer reservoir [17].

In this work, we have explored a nonconventional procedure applied in the control of diffusion of drugs along the wall of electrospun fibers. Using a single fluid for electrospinning process, we have explored the influence of additive (block copolymers of poly(ethylene)-b-poly(ethylene oxide)
(PE-b-PEO)) on the overall response of fibers, in order to improve the transit time of fibers at undesirable $\mathrm{pH}$.

The introduction of reasonable number of parameters (concentration of additive, concentration of enteric polymer, and concentration and type of drug) characterizes an additional difficulty in the analysis of data, due to the interaction of different parameters on overall response of systems. In order to overcome this limitation, factorial experimental planning has been considered as a traditional statistical tool for identification of most important parameters.

Concentration of Eudragit L-100 for electrospinning procedure was varied in order to estimate the influence of amount of carrier on diameter of resulting fiber while concentration of drug (nifedipine) and additive (PE-b-PEO block copolymers) were varied in order to control the adequate behavior of drug release at $\mathrm{pH} 6.8$.

The importance of specific parameter is given by average of differences between media of response of samples prepared using the larger and smaller concentration designed for each parameter. If we considered the concentration of released drug as a response, a positive value in the importance of factor reveals that parameter under analysis provides a fast release of drug at fixed interval of time. Using the value of importance of factor, we can define the relative order of importance of each parameter according its potential in the control of characteristic time for drug release.

In order to facilitate the description of systems, each sample was identified as a composition of letters in which the presence of each one characterizes corresponding maximum value of parameter; for example, sample ENP is prepared with maximum in the concentration of Eudragit L-100 (E), nifedipine $(\mathrm{N})$, and PE-b-PEO (P).

The importance of factor [Eudragit L-100] (defined in (1)) characterizes the difference of average of amount of released drug in formulations in which maximum and minimum of Eudragit L-100 are considered (see corresponding values for each sample in the right side of Table 1):

$$
\begin{aligned}
{[\text { Eudragit L-100] }=} & \frac{1}{4}(\mathrm{E}+\mathrm{EN}+\mathrm{ENP}+\mathrm{EP}) \\
& -\frac{1}{4}(\mathrm{I}+\mathrm{P}+\mathrm{N}+\mathrm{NP})
\end{aligned}
$$

The importance of factor [PE-b-PEO] on controlled release of drug is given by

$$
\begin{aligned}
{[\mathrm{PE}-\mathrm{b}-\mathrm{PEO}]=} & \frac{1}{4}(\mathrm{P}+\mathrm{NP}+\mathrm{ENP}+\mathrm{EP}) \\
& -\frac{1}{4}(\mathrm{I}+\mathrm{E}+\mathrm{N}+\mathrm{EN}) .
\end{aligned}
$$

Meanwhile, the importance of parameter [Nifedipine] is given by

$$
\begin{aligned}
{[\text { Nifedipine }]=} & \frac{1}{4}(\mathrm{NP}+\mathrm{ENP}+\mathrm{NE}+\mathrm{N}) \\
& -\frac{1}{4}(\mathrm{I}+\mathrm{E}+\mathrm{P}+\mathrm{EP}) .
\end{aligned}
$$


TABLE 1: Concentration of released nifedipine from electrospun fibers obtained at different combination of parameters (E, N, and P).

\begin{tabular}{lcccc}
\hline Samples & Eudragit L- $100(\mathrm{E})(\mathrm{mg} / \mathrm{mL})$ & Nifedipine $(\mathrm{N})(\mathrm{mg})$ & PE-b-PEO $(\mathrm{P})(\mathrm{mg})$ & {$[$ Released nifedipine] $(\mu \mathrm{g} / \mathrm{mL})$} \\
\hline I & 200 & 100 & 0 & 64.31 \\
P & 200 & 100 & 100 & 47.77 \\
N & 200 & 200 & 0 & 80.31 \\
NP & 200 & 200 & 100 & 49.84 \\
E & 230 & 100 & 0 & 66.46 \\
EP & 230 & 100 & 100 & 27.07 \\
EN & 230 & 200 & 0 & 69.82 \\
ENP & 230 & 200 & 100 & 31.19 \\
\hline
\end{tabular}

\section{Materials and Methods}

2.1. Samples Preparation. Eudragit L-100 (Evonik), nifedipine (Vetec), and poly(ethylene)-b-poly(ethylene oxide) (PE-bPEO) with $20 \%$ of PEO $\left(\left(-\mathrm{CH}_{2}-\mathrm{CH}_{2}\right)_{80}-\left(-\mathrm{CH}_{2}-\mathrm{CH}_{2}-\mathrm{O}\right)_{20}\right.$ $\left.\mathrm{CH}_{2} \mathrm{CH}_{2} \mathrm{OH}\right)$, Aldrich, $\mathrm{Mn}=875 \mathrm{~g} / \mathrm{mol}$, were used as received.

Alcoholic solutions of carrier polymer (Eudragit L-100) were prepared from inclusion of $0.9-1.6 \mathrm{~g}$ of enteric polymer in $6 \mathrm{~mL}$ of ethylic alcohol at $25^{\circ} \mathrm{C}$ and mechanically stirred until complete dispersion of polymer. For statistical analysis, three different measurements were provided for each sample, prepared in triplicate.

2.2. Experimental Setup for Electrospinning Deposition. Resulting solution was disposed in a tubular compartment of $6 \mathrm{~mL}$ connected to a metallic needle and maintained under fixed pressure in a flux of $166 \mu \mathrm{L} / \mathrm{min}$. Then, an electrical excitation from a high voltage source $(15 \mathrm{kV})$ was switched on and a resulting electric field was established between the tip of needle and a grounded target (separated by $10 \mathrm{~cm}$ ). The sample holder (a metallic flat surface disposed on the surface of a grounded target plane) collected the ejected fibers in fixed interval of time of five minutes.

The incorporation of drug was established after complete dispersion of active probe molecules (nifedipine 100-200 mg) into Eudragit L-100 alcoholic solution under mechanical stirring.

2.3. SEM Images and Statistical Analysis. SEM images were performed from the use of two different microscopies (Hitachi TM 1000 and Vega 3XM Tescan). We have determined the fiber diameter size from the use of nine different images of SEM for each sample. Distribution of fiber diameter was statistically calculated using the software Minitab 14 (statistics package) and ImageJ (public-domain image processing and analysis program developed at US National Institute of Health (NIH)) which was used for calculus of average fiber diameter $(\bar{d})$ distribution per image.

\subsection{Differential Scanning Calorimetry and PXRD Analysis.} DSC thermograms of drug-loaded fibers were performed by a Shimadzu DSC-60 in order to evaluate the influence of additives on response of system. We have used a scan rate of $10^{\circ} \mathrm{C} / \mathrm{min}$ and a range of temperature of $25^{\circ} \mathrm{C}$ to $280^{\circ} \mathrm{C}$ under continuous flux of nitrogen. Powder X-Ray Diffraction (PXRD) was provided by a DRX Equinox 1000, Inel.

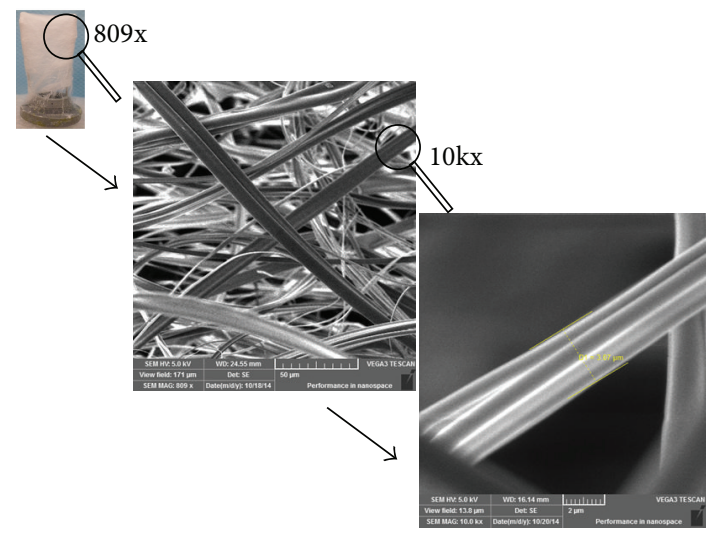

FIgURE 1: SEM images of electrospun fibers of Eudragit L-100.

2.5. FTIR and Profile of Drug Release. FTIR analysis was performed using an IR Prestige-21 FTIR Shimadzu while drug delivery profile was determined in an UV-Vis spectrophotometer Hach DR 5000.

2.6. Release Study. For measurement of controlled release of drug, $50 \mathrm{mg}$ of fibers was dispersed into simulated GIT fluid (Intestinal Fluid, Simulated, TS, according to procedure established at USP 36-Reagents) and maintained at continuous stirring $(90 \mathrm{rpm})$ in an orbital shaker. Aliquots of resulting solution were analyzed in an UV-vis spectrophotometer during fixed interval of time and intensity of characteristic peak of drug was explored for measurement of kinetics of release.

\section{Results and Discussion}

An important aspect to be preliminarily reported is relative to the negligible density of beads in the collected fibers of Eudragit L-100 at different relative concentration. The morphology of resulting fibers is shown in Figure 1. Added to the rare imperfections along the net, the diameter of fibers strongly depends on the concentration of enteric polymer.

3.1. Influence of Concentration of Eudragit L-100 on Diameter of Electrospun Fibers. The influence of relative concentration of Eudragit L-100 on diameter of resulting electrospun fibers was analyzed in terms of diameter of fibers in SEM images, as summarized in Figure 2. As we can see, the increase 

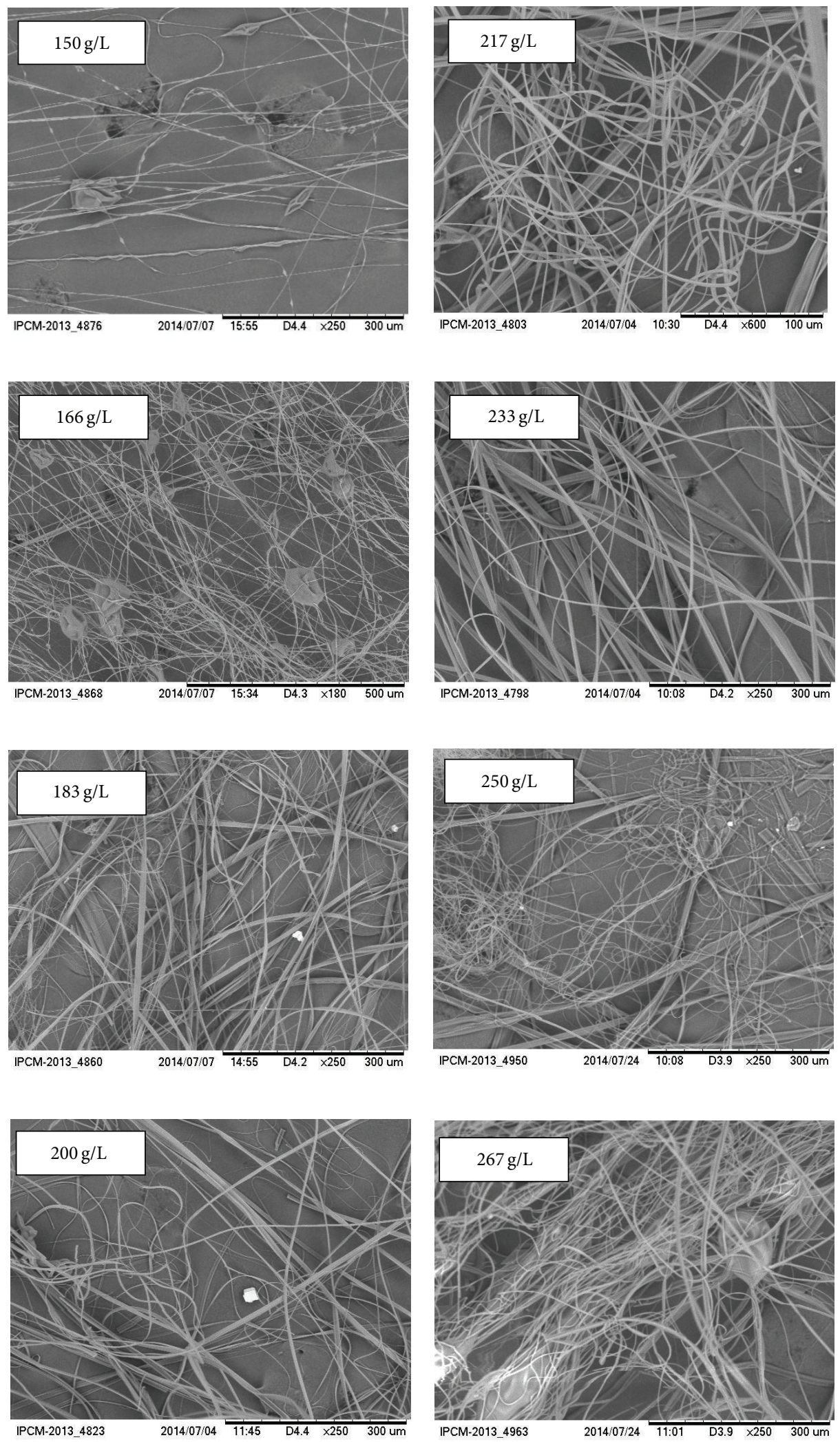

Figure 2: Comparison of electrospun fibers of Eudragit L-100 as a function of concentration of polymer (range of $150 \mathrm{~g} / \mathrm{L}$ to $267 \mathrm{~g} / \mathrm{L}$ ). 


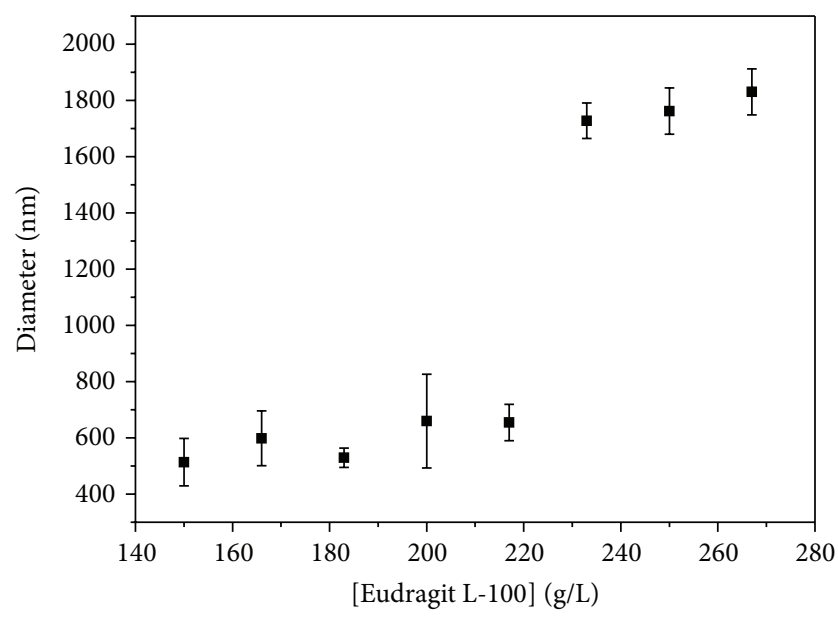

FIGURE 3: Dependence of diameter of electrospun fibers versus concentration of Eudragit L-100.

in the relative concentration of enteric polymer induces a progressive increase in the diameter of resulting fiber. The dependence of diameter of resulting fiber with concentration of polymeric carrier presents an interesting behavior, as indicated in Figure 3. Different plateaus indicate that a typical transition is established in the diameter of fibers: at relative concentration of Eudragit $C<220 \mathrm{~g} / \mathrm{L}$ (low level), a typical invariance in the diameter of resulting fiber is established (average diameter in order of $500 \mathrm{~nm}$ ). At high concentration of enteric polymer in alcoholic solution $(C>230 \mathrm{~g} / \mathrm{L})$, a new plateau is attained and diameter of fibers tends towards a value in order of $1.7 \mu \mathrm{m}$. These results establish a critical concentration of Eudragit L-100 in which the diameter of fibers tends towards a value in order of three times compared to the initial diameter (low concentration of Eudragit L-100).

3.2. Influence of Additives, Enteric Polymer, and Drug on Controlled Release Provided by Electrospun Fibers. Using the previous information, we have explored two different diameter levels of electrospun fibers of Eudragit L-100 (low $(C<220 \mathrm{~g} / \mathrm{L})$ and high $(C>230 \mathrm{~g} / \mathrm{L}))$ in association with presence/absence of retardant (PE-b-PEO block copolymers) and concentration of drug (high and low concentration of nifedipine). Using extreme levels for three independent variables, we have prepared groups of eight samples and verified the potential for application in drug delivery systems from measurement of absorbance at $238 \mathrm{~nm}$ (characteristic peak of nifedipine) after 10 minutes of immersion in aqueous solution of $\mathrm{NaOH}$ at $\mathrm{pH}$ 6.8. Using a calibration curve of nifedipine (application of Beer-Lambert law), absorbance data was converted into relative concentration of drug in aqueous solution. If we considered the same time for release of all of the systems, the degree of encapsulation can be estimated from comparison of amount of drug released in water as a response of different conditions of preparation.

Table 1 summarizes the results of released nifedipine (in $\mu \mathrm{g} / \mathrm{mL}$ ) at different combination of parameters (Eudragit L100, nifedipine, and PE-b-PEO). The higher concentration of released drug is verified at higher concentration of nifedipine, as expected.

On the other side, the association of high concentration of Eudragit L-100 and PE-b-PEO reduces the amount of released drug (around 38\%) in comparison with corresponding high concentration of nifedipine, allowing the application of PE$\mathrm{b}-\mathrm{PEO}$ as a potential retardant for drug release.

Other important response is obtained from comparison of release profile with high concentration of nifedipine/low concentration of Eudragit-100 and high concentration of both components. As we can see from data of Table 1, the increase in the diameter of fiber contributes with decrease in the amount of released drug at fixed time, since diffusion of drug depends on thickness of enteric layer.

Corresponding value for importance of [Eudragit L-100] in order of -11.92 (result of (1) applied in data of Table 1) indicates that release rate of drug varies inversely with the increase in the diameter of fibers, allowing the prolonged transit time of substance along the wall of thicker polymeric fibers.

In the same direction, the parameter [PE-b-PEO] (2) returns an importance of -31.26 , which characterizes the superior response of additive as a retardant for controlled release of drug.

As expected, the importance of parameter [nifedipine] (3) returns a positive value $(+6.39)$ due to the direct relationship between relative increase in the drug concentration dispersed in polymer carrier and released amount of drug.

The importance of parameter for combined action of variables "interaction effects" results in negative values if we considered the combination of two parameters: [EDGT + Nifedipine $]=-2.65$, $[$ EDGT + PE-b-PEO $]=-7.75$, and [nifedipine + PE-b-PEO] $=-3.29$, since typical response of PE-b-PEO and EDGT dominates on improved release of nifedipine induced by high concentration of drug.

Based on these results, it is possible to verify that combined action of enteric polymer and block copolymer improves the protection of drug against aggressive condition (low pH at stomach).

3.3. Structure of Electrospun Fibers. The FTIR spectra for pure PE-b-PEO, electrospun fibers of Eudragit L-100, and Eudragit L-100/PE-b-PEO are shown in Figure 4.

Characteristic peaks of poly(ethylene) are assigned to asymmetric/symmetric stretching vibration modes of $\mathrm{CH}_{2}$ (2917/2849) $\mathrm{cm}^{-1}$, bending vibration modes of $\mathrm{CH}_{2}$ at $1473 / 1462 \mathrm{~cm}^{-1}$, and rocking vibration mode of $\mathrm{CH}_{2}$ at $719 \mathrm{~cm}^{-1}[18]$.

PEO groups are characterized by stretching vibration modes of COC at $1121 \mathrm{~cm}^{-1}$ and stretching vibration of $\mathrm{OH}$ at $3400 \mathrm{~cm}^{-1}$.

Characteristic peaks of electrospun fibers of Eudragit L100 are $3503 \mathrm{~cm}^{-1}$ (assigned to free form of carboxylic acid) [19], $2998 \mathrm{~cm}^{-1}\left(\mathrm{CH}_{x}\right.$ vibration) [20], and $1726 \mathrm{~cm}^{-1}$ (dimmer vibration of carboxylic ester) and ester vibrations at $1158 \mathrm{~cm}^{-1}$ [19].

The incorporation of PE-b-PEO in the resulting electrospun fibers of Eudragit L-100/PE-b-PEO can be detected from 


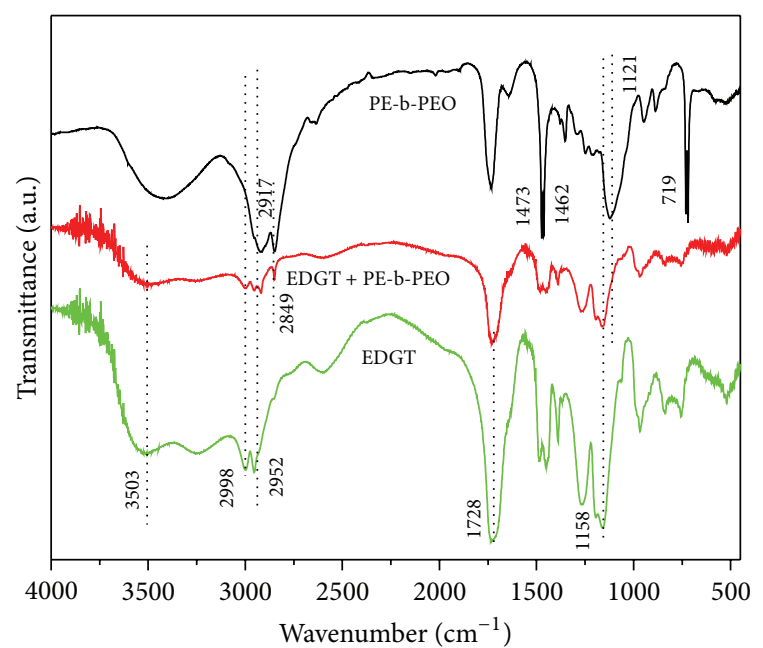

FIGURE 4: FTIR spectrum of pristine PE-b-PEO and electrospun fibers of Eudragit L-100 (EDGT) and Eudragit L-100 + PE-b-PEO $(\mathrm{EDGT}+\mathrm{PE}-\mathrm{b}-\mathrm{PEO})$.

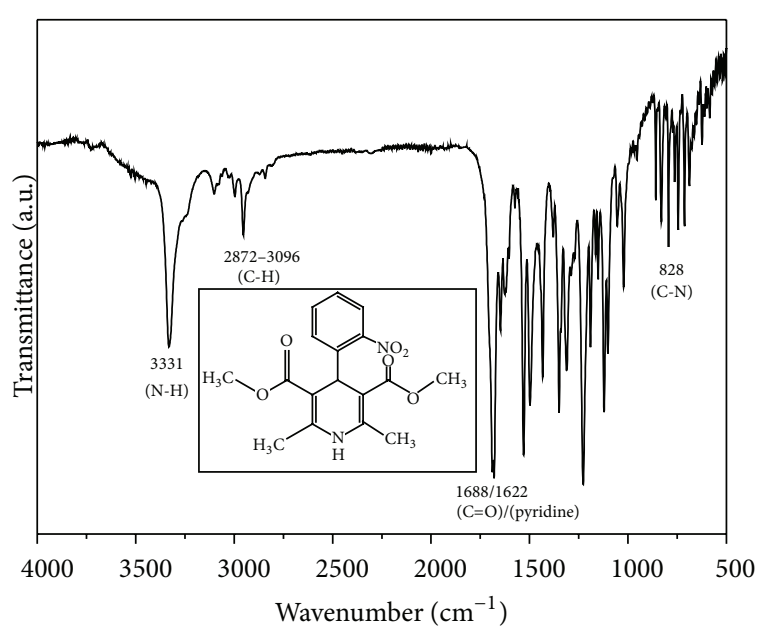

FIGURE 5: FTIR spectrum of pristine nifedipine.

the presence of additional peaks in the FTIR spectrum of hybrid fibers such as at $2917 / 2849 \mathrm{~cm}^{-1}$, because the reasonable degree of dispersion of block copolymers is established along the produced fibers.

The structure of encapsulated drug (nifedipine) is detailed in the FTIR of Figure 5. As we can see, characteristic groups of molecule are present in the spectrum [21, 22], and molecules are preserved during preparation of electrospun fibers.

PXRD pattern of pristine nifedipine exhibits sharp peaks at $2 \theta$ angles of $7.3,9.6,10.9,13.8,18.6,21.4,23.7$, and 26.8 [21, 23] (as shown in Figure 6) characterizing the use of crystalline nifedipine during electrospinning encapsulation.

The degree of encapsulation and integrity of drugs after release from hybrid polymeric matrix were analyzed from comparison of UV-vis spectrum of free molecules dispersed in simulated GIT solution and after the controlled release.

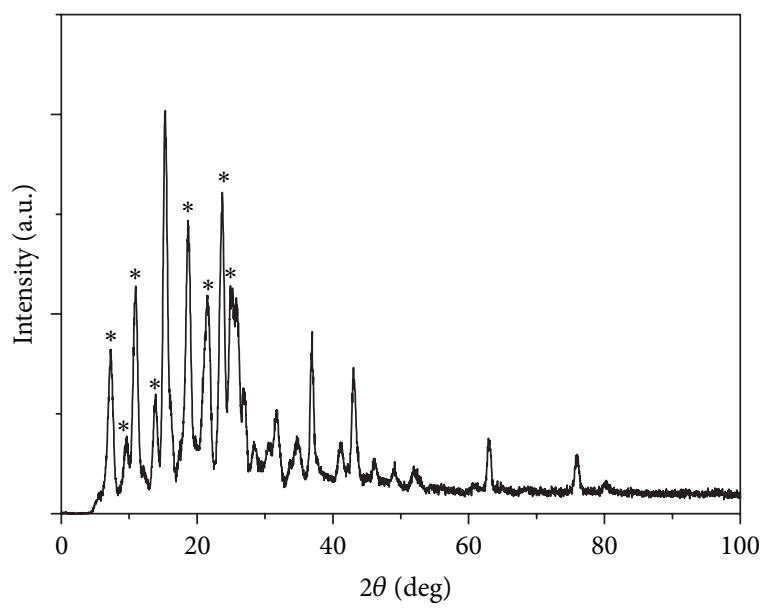

FIgURe 6: Powder XRD of pristine nifedipine.

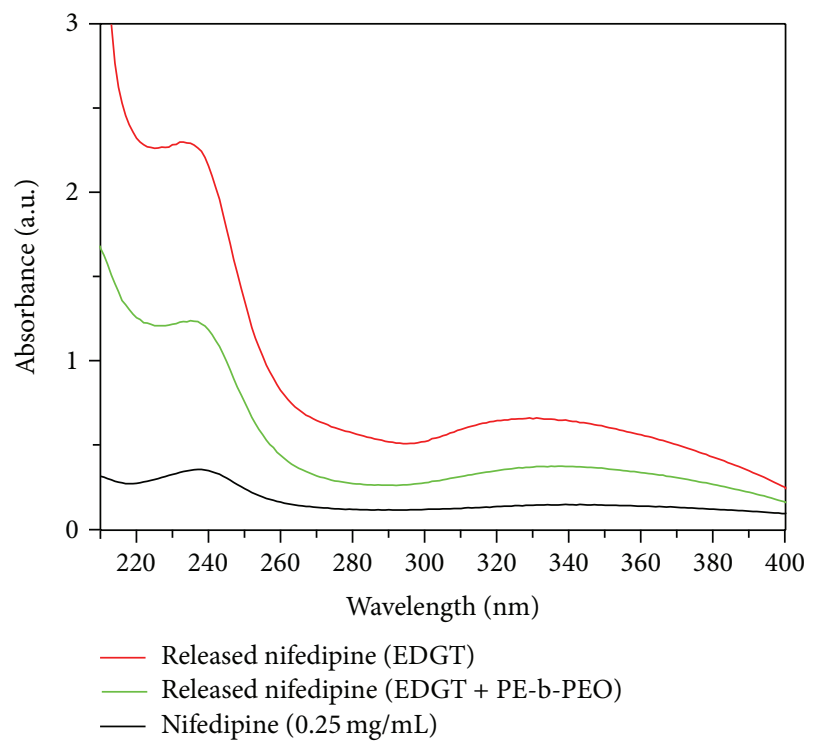

FIGURE 7: UV-vis spectrum of pristine nifedipine and released drug from electrospun fibers of Eudragit L-100 and Eudragit L-100 + PEb-PEO.

As we can see in Figure 7, released drug presents equivalent peaks in comparison with pristine ones, which is strong evidence that encapsulation preserves the chemical structure of drug and influence of light (photodegradation of drug) can be considered as negligible.

DSC thermograms of electrospun fibers of Eudragit L-100 + nifedipine, Eudragit L-100 + PE-b-PEO + nifedipine, PE-bPEO, nifedipine, and Eudragit L-100 are shown in Figure 8. As reported in the literature $[24,25]$, characteristic endothermic peak of Eudragit L-100 at $210^{\circ} \mathrm{C}$ is attributed to the loss of water due to the anhydride formation [24]. Characteristic peak relative to the melting point of pure nifedipine (sharp peak at $171.3^{\circ} \mathrm{C}$, as shown in Figure 8) [26] indicates the typical signature of drug.

The incorporation of nifedipine and PE-b-PEO in the structure of electrospun fibers of Eudragit L-100 promotes 


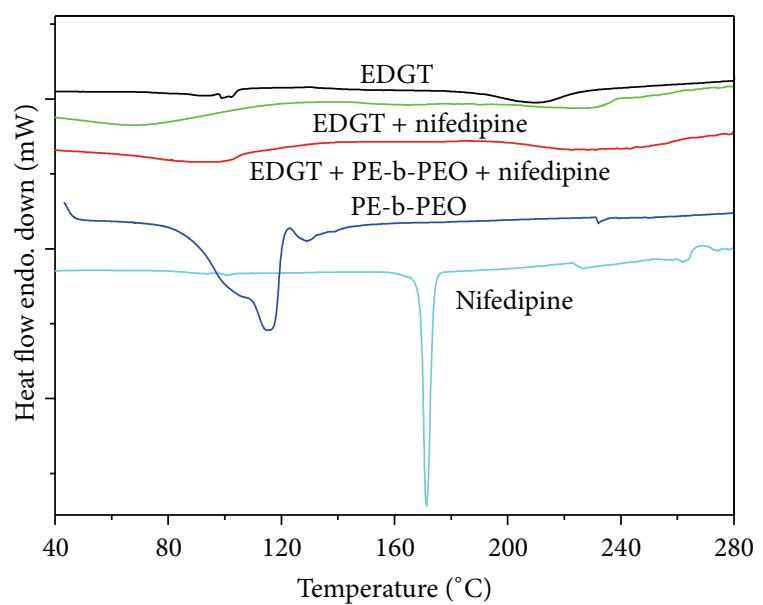

Figure 8: Comparison of DSC thermograms of Eudragit, PE-b$\mathrm{PEO}, \mathrm{EDGT}+$ nifedipine, EDGT + PE-b-PEO + nifedipine, and pristine nifedipine.

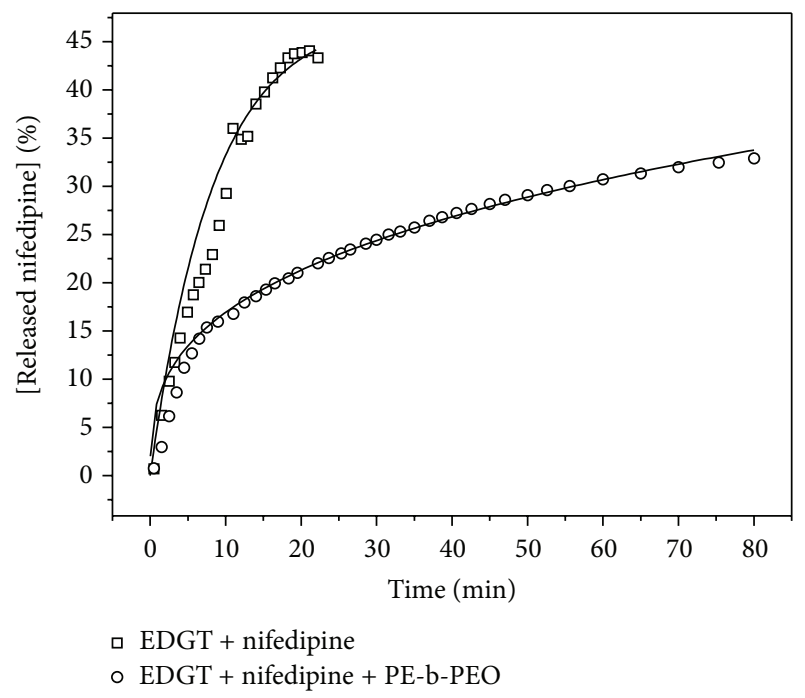

FIgURE 9: Comparison of release profile of nifedipine encapsulated by Eudragit L-100 and Eudragit L-100 + PE-b-PEO.

a slight shift in the characteristic temperature and a broader endothermic peak for resulting material.

3.4. Controlled Release Analysis. The kinetics of controlled release of nifedipine was explored in the presence and absence of retardant (PE-b-PEO).

As we can see in Figure 9, the introduction of PE-b-PEO into electrospun fibers affects strongly the kinetics of drug release, allowing the prolonged release of drug at $\mathrm{pH} 6.8$.

According to Kosmidis et al. [27], important modifications on release profile are induced by geometry of matrix and diffusion of drug along cylindrical matrices is conveniently described by Weibull function, given by

$$
\frac{M_{t}}{M_{\infty}}=1-\exp \left(-a t^{b}\right)
$$

where $a$ represents scale parameter relative to the time dependence while $b$ represents the dissolution curve progression. The release from electrospun fibers of Eudragit L-100 returned a value of $b=1$, and the resulting curve (shown as a continuous line) has a typical exponential profile for release as a response of rapid release of drug.

On the other side, the introduction of PE-b-PEO in the cylindrical matrix affects the kinetics of release and a typical power law $M_{t} / M_{\infty}=k t^{n}$ (Korsmeyer-Peppas model) with a value of $n=0.3$ describes the behavior of controlled release of nifedipine in the presence of polymeric micelles of PEb-PEO. Solid lines in Figure 9 indicate a good agreement between fitting and experimental data.

The incorporation of nifedipine into the core of PE-bPEO polymeric micelles creates hydrophobic nanoenvironment dispersed into Eudragit L-100 matrix with homogeneous distribution of drug into enteric matrix. This process contributes with a more homogeneous diffusion of drug along fibers and subsequent prolonged action during controlled release of drug. If we considered that $49.63 \mathrm{mg}$ of drug is effectively loaded into Eudragit L-100 matrix and electrospun deposition of $6 \mathrm{~mL}$ of solution results in the production of $259.2 \mathrm{mg}$ of fibers, we can establish that relative concentration of drug per fiber is $19.14 \mathrm{wt} \%$. After controlled release of drug into GIT fluid, a typical concentration of drug, $51.9 \mu \mathrm{g} / \mathrm{mL}$, is attained after 20 minutes (absence of PEb-PEO), with $43.3 \%$ of drug available in aqueous solution during initial minutes of release.

\section{Conclusions}

The development of single solution for electrospun production of drug carriers can be strongly improved from introduction of additives such as block copolymers of PEb-PEO which contributes with retarding effects of release in critical situation, namely, extremely high $\mathrm{pH}$, in which the solubility of Eudragit L-100 takes place. The incorporation of additives of low cost in association with mass production of electrospun single core fibers represents a promising procedure to be applied in the production of low cost carriers for colonic action of diverse drugs. The balance between relative concentration of enteric polymer matrix and concentration of additive applied during electrospinning introduces a critical parameter in the definition of controlled release time of encapsulated drug.

\section{Conflict of Interests}

The authors declare that there is no conflict of interests regarding the publication of this paper.

\section{References}

[1] B. Li, J. He, D. G. Evans, and X. Duan, "Enteric-coated layered double hydroxides as a controlled release drug delivery system," International Journal of Pharmaceutics, vol. 287, no. 1-2, pp. 8995, 2004.

[2] F. Siepmann, A. Hoffmann, B. Leclercq, B. Carlin, and J. Siepmann, "How to adjust desired drug release patterns 
from ethylcellulose-coated dosage forms," Journal of Controlled Release, vol. 119, no. 2, pp. 182-189, 2007.

[3] M. Rodríguez, J. L. Vila-Jato, and D. Torres, "Design of a new multiparticulate system for potential site-specific and controlled drug delivery to the colonic region," Journal of Controlled Release, vol. 55, no. 1, pp. 67-77, 1998.

[4] M. Z. I. Khan, Ž. Prebeg, and N. Kurjaković, "A pH-dependent colon targeted oral drug delivery system using methacrylic acid copolymers. I. Manipulation of drug release using Eudragit L100-55 and Eudragit S100 combinations," Journal of Controlled Release, vol. 58, no. 2, pp. 215-222, 1999.

[5] C. Damgé, P. Maincent, and N. Ubrich, "Oral delivery of insulin associated to polymeric nanoparticles in diabetic rats," Journal of Controlled Release, vol. 117, no. 2, pp. 163-170, 2007.

[6] M. Ashford, J. T. Fell, D. Attwood, and P. J. Woodhead, "An in vitro investigation into the suitability of $\mathrm{pH}$-dependent polymers for colonic targeting," International Journal of Pharmaceutics, vol. 91, no. 2-3, pp. 241-245, 1993.

[7] H. P. de Oliveira, J. J. F. Albuquerque Jr., C. Nogueiras, and J. Rieumont, "Physical chemistry behavior of enteric polymer in drug release systems," International Journal of Pharmaceutics, vol. 366, no. 1-2, pp. 185-189, 2009.

[8] H. P. de Oliveira, G. F. Tavares, C. Nogueiras, and J. Rieumont, "Physico-chemical analysis of metronidazole encapsulation processes in Eudragit copolymers and their blending with amphiphilic block copolymers," International Journal of Pharmaceutics, vol. 380, no. 1-2, pp. 55-61, 2009.

[9] A. Akhgari, Z. Heshmati, and B. S. Makhmalzadeh, "Indomethacin electrospun nanofibers for colonic drug delivery: preparation and characterization," Advanced Pharmaceutical Bulletin, vol. 3, no. 1, pp. 85-90, 2013.

[10] V. V. Sokolov, N. R. Kildeeva, I. Y. Filatov, and Y. N. Filatov, "Development of a locally anesthetizing wound dressing based on eudragit RS ultrathin fibers," Fibre Chemistry, vol. 45, no. 2, pp. 74-78, 2013.

[11] E. S. Araújo, M. L. F. Nascimento, and H. P. de Oliveira, "Influence of triton X-100 on PVA fibres production by the electrospinning technique," Fibres \& Textiles in Eastern Europe, vol. 4, no. 100, pp. 39-43, 2013.

[12] K. Karthikeyan, S. Guhathakarta, R. Rajaram, and P. S. Korrapati, "Electrospun zein/eudragit nanofibers based dual drug delivery system for the simultaneous delivery of aceclofenac and pantoprazole," International Journal of Pharmaceutics, vol. 438, no. 1-2, pp. 117-122, 2012.

[13] X. Shen, D. Yu, L. Zhu, C. Branford-White, K. White, and N. P. Chatterton, "Electrospun diclofenac sodium loaded Eudragit L 100-55 nanofibers for colon-targeted drug delivery," International Journal of Pharmaceutics, vol. 408, no. 1-2, pp. 200-207, 2011.

[14] D.-G. Yu, Y. Xu, Z. Li, L.-P. Du, B.-G. Zhao, and X. Wang, "Coaxial electrospinning with mixed solvents: from flat to round eudragit L100 nanofibers for better colon-targeted sustained drug release profiles," Journal of Nanomaterials, vol. 2014, Article ID 967295, 8 pages, 2014.

[15] Z. Xiang, P. Sarazin, and B. D. Favis, "Controlling burst and final drug release times from porous polylactide devices derived from co-continuous polymer blends," Biomacromolecules, vol. 10, no. 8, pp. 2053-2066, 2009.

[16] A. Rösler, G. W. M. Vandermeulen, and H.-A. Klok, "Advanced drug delivery devices via self-assembly of amphiphilic block copolymers," Advanced Drug Delivery Reviews, vol. 64, pp. 270279, 2012.
[17] M. L. Adams, A. Lavasanifar, and G. S. Kwon, "Amphiphilic block copolymers for drug delivery," Journal of Pharmaceutical Sciences, vol. 92, no. 7, pp. 1343-1355, 2003.

[18] X. Zheng, Q. Xu, L. He et al., "Modification of graphene oxide with amphiphilic double-crystalline block copolymer polyethylene-b-poly(ethylene oxide) with assistance of supercritical $\mathrm{CO}_{2}$ and its further functionalization," The Journal of Physical Chemistry B, vol. 115, no. 19, pp. 5815-5826, 2011.

[19] T. M. M. Santos, P. H. Oliveira Jr., L. A. A. Ribeiro, and H. P. de Oliveira, "Drug/magnetite-loaded enteric particles: the influence of localized magnetic field on controlled release of nifedipine," Asian Journal of Biochemical and Pharmaceutical Research, vol. 4, no. 1, pp. 63-71, 2014.

[20] M. Bharathi, S. C. Prasad, R. L. Eswari et al., "Preparation and in vitro \& in vivo characterization of valsartan loaded eudragit nanoparticles," Der Pharmacia Sinica, vol. 3, no. 5, pp. 516-525, 2012.

[21] Y. Lalitha and P. K. Lakshmi, "Enhancement of dissolution of nifedipine by surface solid dispersion technique," International Journal of Pharmacy and Pharmaceutical Sciences, vol. 3, no. 3, pp. 41-46, 2011.

[22] N. Kanagathara, P. Shenbagarajan, C. E. Jeyanthi, and M. Thirunavukkarasu, "Fourier transform infrared spectroscopic investigation on nifedipine," International Journal of Pharmacy and Biological Sciences, vol. 1, pp. 52-56, 2011.

[23] T. Sharkawi, E. Ruiz, T. Cacciaguerra, M. Domurado, and B. Bataille, "Preliminary investigation of improved solubility of nifedipine by co processing with vinylcaprolactam/vinylacetate/PEG6000 copolymer through spray dried solid dispersions," Macromolecular Symposia, vol. 336, no. 1, pp. 47-52, 2014.

[24] S.-Y. Lin, C.-M. Liao, and G.-H. Hsiue, "Temperaturedependent anhydride formation of Eudragit L-100 films determined by reflectance FTi.r./d.s.c. microspectroscopy," Polymer, vol. 36, no. 16, pp. 3239-3241, 1995.

[25] M. Sharma, V. Sharma, A. K. Panda, and D. K. Majumdar, "Enteric microsphere formulations of papain for oral delivery," Yakugaku Zasshi, vol. 131, no. 5, pp. 697-709, 2011.

[26] D. Grooff, M. M. de Villiers, and W. Liebenberg, "Thermal methods for evaluating polymorphic transitions in nifedipine," Thermochimica Acta, vol. 454, no. 1, pp. 33-42, 2007.

[27] K. Kosmidis, P. Argyrakis, and P. Macheras, "A reappraisal of drug release laws using Monte Carlo simulations: the prevalence of the Weibull function," Pharmaceutical Research, vol. 20, no. 7, pp. 988-995, 2003. 

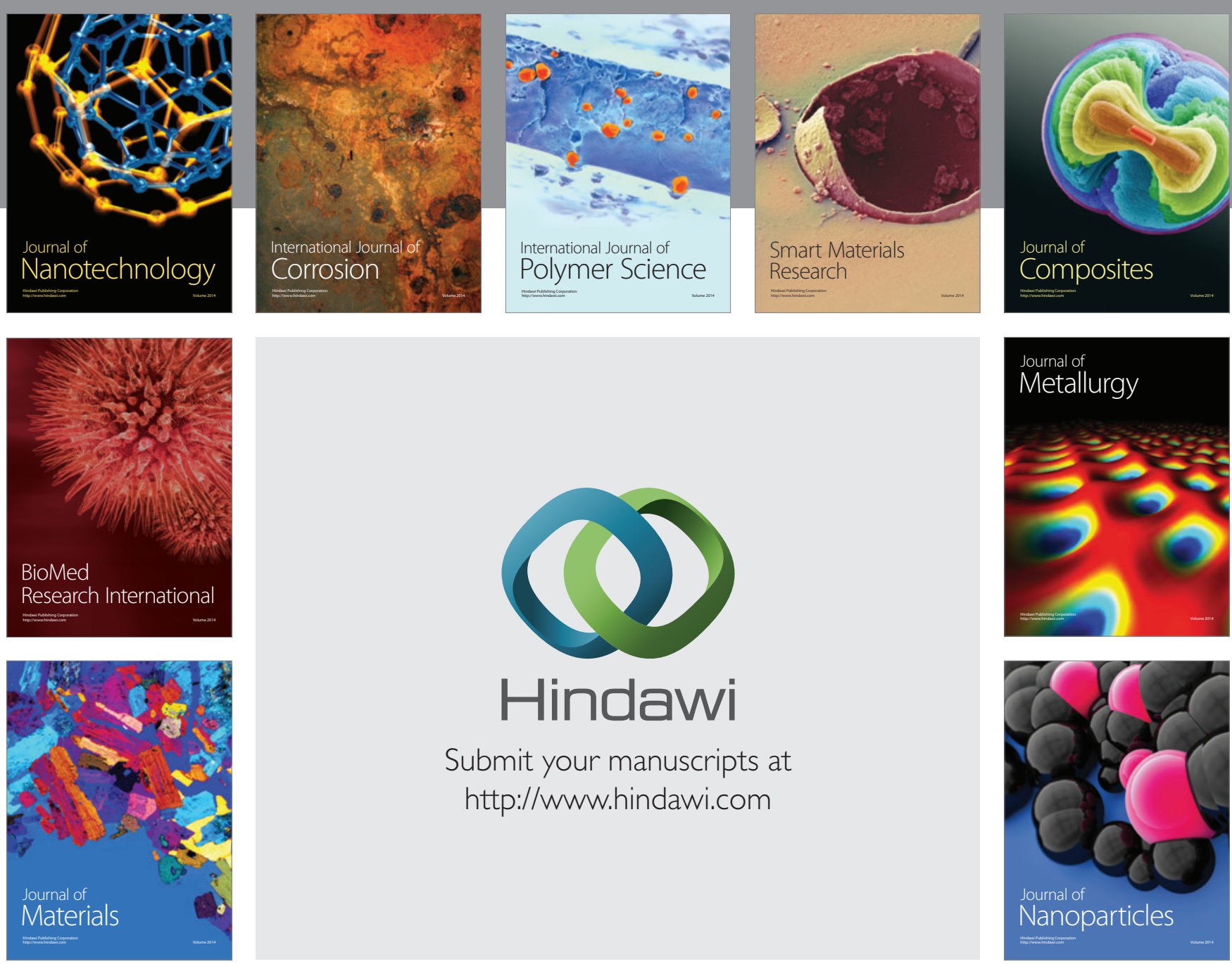

Submit your manuscripts at http://www.hindawi.com
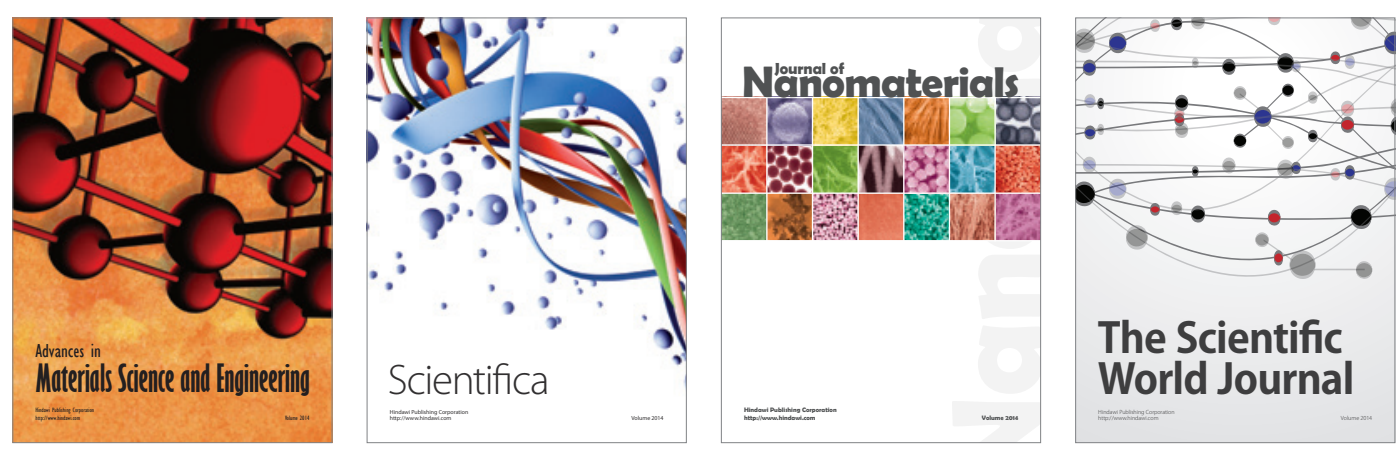

\section{The Scientific World Journal}
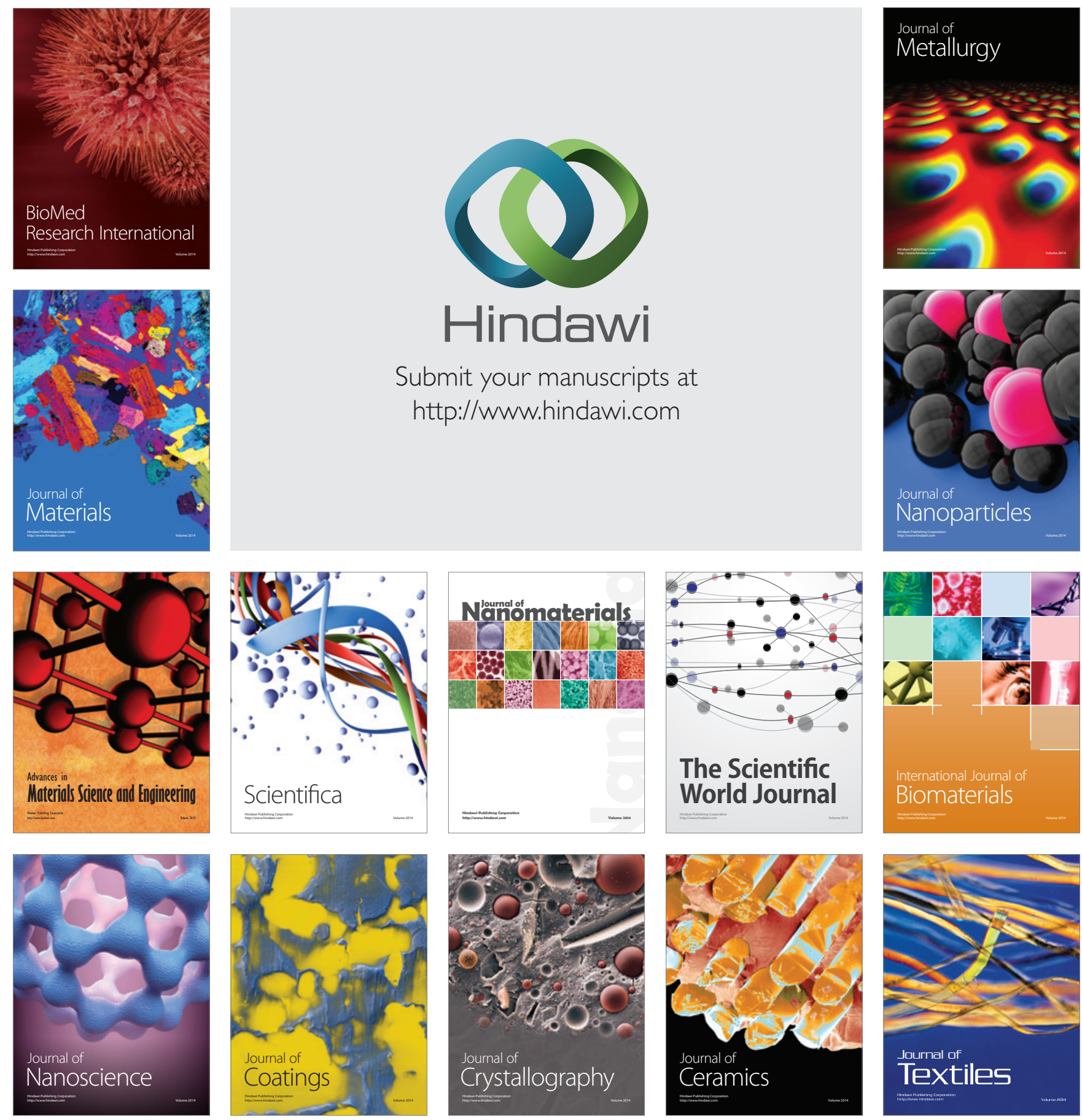